\title{
SESSION IV
}

\author{
Chairman: DR E. G. J. OLSEN
}

\section{A comparison of the clinical and cardiological features of endomyocardial disease in temperate and tropical regions}

\author{
J. DAVIES* \\ M.R.C.P. \\ C. J. F. SPRY \\ M.R.C.P., D. Phil., M.R.C.Path. \\ G. VIJAYARAGHAVAN $\dagger$ \\ M.D., D.M. \\ J. A. DE SOUZA \\ M.D.
}

${ }^{*}$ Department of Immunology, Royal Postgraduate Medical School, London, †Medical College Hospital, Trivandrum, Kerala, India and $\ddagger$ University Hospital, Bahia, Brazil

\section{Summary}

This study was designed to compare the clinical and cardiological features of endomyocardial disease in temperate and tropical regions. Eleven patients were studied in the U.K., 47 in India and 8 in Brazil. The patients in the U.K. were older, with a male predominance, and they had a systemic illness: the hypereosinophilic syndrome. Half of these patients presented in the early necrotic stage of the disease, and all had biventricular involvement. On the other hand, patients in the tropical countries were younger, with an equal sex incidence, and were from poor, malnourished communities with heavy parasite loads, especially filariasis in India. None presented in the early necrotic stage of the disease and a quarter had isolated right or left ventricular disease.

In order to account for these differences between patients in temperate and tropical regions with endomyocardial disease, it was proposed that the nature of the underlying disease and the rate at which endomyocardial lesions develop, determine the clinical features of this disorder. In temperate climates eosinophil granule toxins may produce a rapidly progressive form of the disease in patients with the hypereosinophilic syndrome, whereas the disease may take longer to develop in patients in tropical climates, who have a less marked eosinophilia due to parasitic infections.

KEY WORDS: endomyocardial disease, temperate, tropical regions.

\section{Introduction}

Tropical endomyocardial disease became widely known after its description in Uganda (Davies, 1948). Subsequent work there clarified the clinical and pathological features of the disorder, and it was classified as a distinct cardiomyopathy (Patel, D'Arbela and Somers, 1977). However, the presence of an eosinophilia in many of these patients, especially those living in areas of endemic filariasis, raised the possibility that tropical endomyocardial disease had a similar pathogenesis to eosinophilic endomyocardial disease, which is mainly seen in temperate climates (Gerbaux et al., 1956; Ive, Willis and Ikeme, 1967). This led Brockington and Olsen (1973) to carry out a review of pathological specimens of late stage (fibrotic) endomyocardial disease which had been recognised in tropical and temperate regions. They found that the lesions were indistinguishable.

During the past 7 years we have been studying patients in the United Kingdom (U.K.) with eosinophilic endomyocardial disease which occurs as one complication of the hypereosinophilic syndrome (Spry et al., 1983). Clinical and experimental studies supported the suggestion that this form of endomyocardial disease was related to the presence of large numbers of eosinophils in the blood, particularly degranulated eosinophils. It is proposed that the granule products from these cells cause the endomyocardial lesions (Spry, Tai and Davies, 1983).

Although it was felt that both tropical and eosinophilic endomyocardial disease could have a similar pathogenesis (Olsen and Spry, 1979), it became necessary to explain why a number of differences had been reported. For this reason it was decided to carry out a study of these forms of endomyocardial disease in the U.K. and tropics, to compare the clinical and cardiological features of endomyocardial disease in these regions. Unfortunately, this could not be done in Uganda, so two 
other tropical regions with a high incidence of tropical endomyocardial fibrosis were chosen: Kerala (South India) and Bahia (Brazil).

\section{Patients}

The 11 patients in the U.K. with eosinophilic endomyocardial disease have been described previously (Davies et al., 1983). They all had biventricular endomyocardial disease. Nine had marked mitral valve disease, and 6 had severe tricuspid regurgitation. Four patients were studied during the early necrotic stage of the disease, and 5 were studied in the late fibrotic stage.

Forty-seven patients were studied in India and 8 patients in Brazil. These patients all had clinical and cardiological features of advanced endomyocardial disease in the late fibrotic stage.

\section{Results}

A summary of the general and cardiovascular features of patients with endomyocardial disease in the temperate region and the two tropical regions is shown in Fig. 1 and 2.

A number of important differences were found. Many patients living in the U.K. were in their fourth decade (mean age 38 years) and 9 were male. Half of them presented in the early necrotic stage of the disease. They all had a systemic illness with hypereo $z$ sinophilia, the hypereosinophilic syndrome. Thi syndrome is associated with wide-spread tissue injury. (Spry, 1982) including the heart (eosinophilic endo myocardial disease), skin, retina (Chaine et al., 1982) blood vessels, lymph nodes, spleen, gastrointestina? tract and lungs. On the other hand, patients in the tropical regions were younger (mean age 17 years i:f India and 30 years in Brazil), and had an equal se $\$$ incidence of the disease. They were from the poores socio-economic groups, with malnutrition and features of chronic growth retardation. They all pre? sented at a late stage of the disease with ascites and/or periorbital oedema. Despite clinical feature of advanced cardiac disease, they were remarkably free from severe symptoms, and appeared to have adapted to their restrictive heart failure. This sugic gested that heart disease had been present for manys years. None of them had suffered from a systemi\&o illness with similarities to the hypereosinophilie syndrome.

Cardiological studies showed some similarities and some differences in the clinical features of patientswith endomyocardial disease from the U.K. and the tropics. Most patients had a loud third heart sounक and raised jugular venous pressure. A positive Kussmaul's sign was noted frequently in both grou $8 \mathrm{sc0}$ However, murmurs of atrio-ventricular valve reger

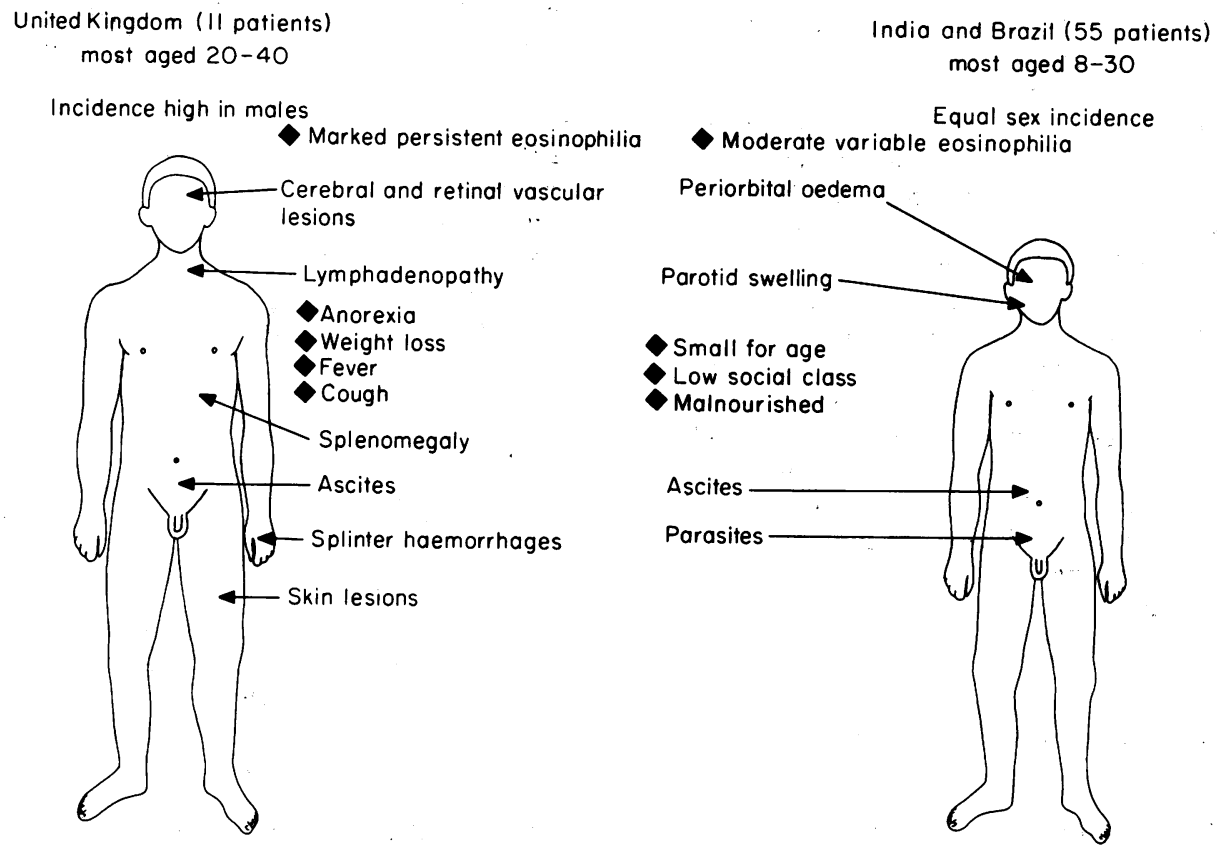

FIG. 1. Comparison of the general clinical features of patients with endomyocardial disease in the U.K. and in India and Brazil. Striking differences are shown which suggest that patients in temperate regions have a more aggressive and rapidly progressive disorder than patients $\overline{0}$ tropical regions. 


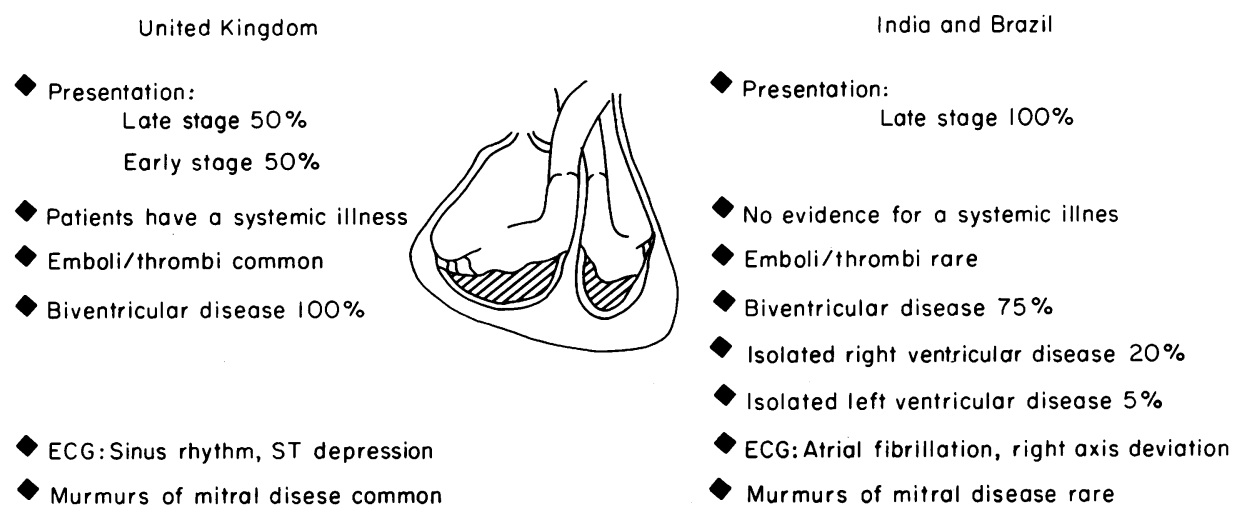

The pathology of late stage disease is identical in both regions

FIG. 2. Comparison of the cardiovascular disease in patients with endomyocardial disease in the U.K. and in India and Brazil. The cardiological features showed many similarities. Differences were largely related to variations in the staging of the disease at diagnosis, and the extent of involvement of right and/or left ventricles. (ECG $=$ electrocardiogram)

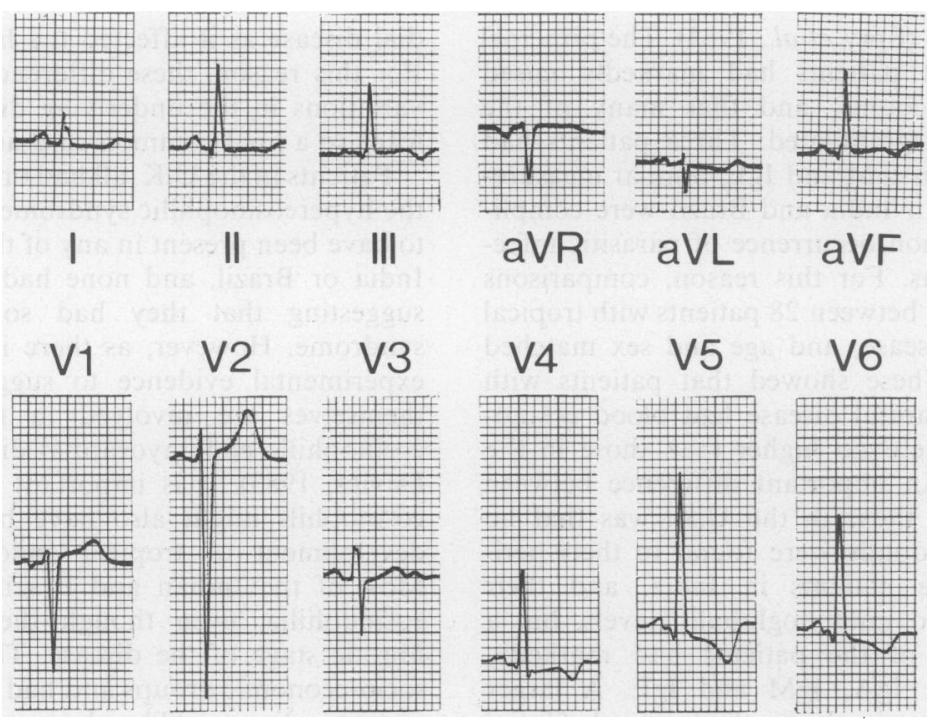

FIG. 3. Electrocardiogram of a man in the U.K. with eosinophilic endomyocardial disease. showing sinus rhythm with widespread ST segment and $T$ wave changes.

gitation, particularly mitral regurgitation, were more common in patients in the U.K.; half of the patients in India and Brazil had no cardiac murmurs, even though they all had severe restrictive heart defects. Electrocardiograms showed some differences: in the U.K. there was sinus rhythm with ST depression (Fig. 3), whereas in India and Brazil atrial fibrillation with right axis deviation was common (Fig. 4).

Echocardiography showed no significant differences between the 2 groups of patients, when staging of the disease was taken into account. Details of the echocardiographic features of eosinophilic endomyo- cardial disease have already been published (Davies et al., 1982). M-mode echocardiography showed nonspecific changes which did not correlate either with the severity of endomyocardial disease or the thickness of the posterior left ventricular wall. On the other hand, two-dimensional echocardiography, and regional echo amplitude analysis, showed abnormalities corresponding to areas of endomyocardial disease.

Results of haematological studies and immunoglobulin measurements in patients with eosinophilic endomyocardial disease in the U.K., have been 


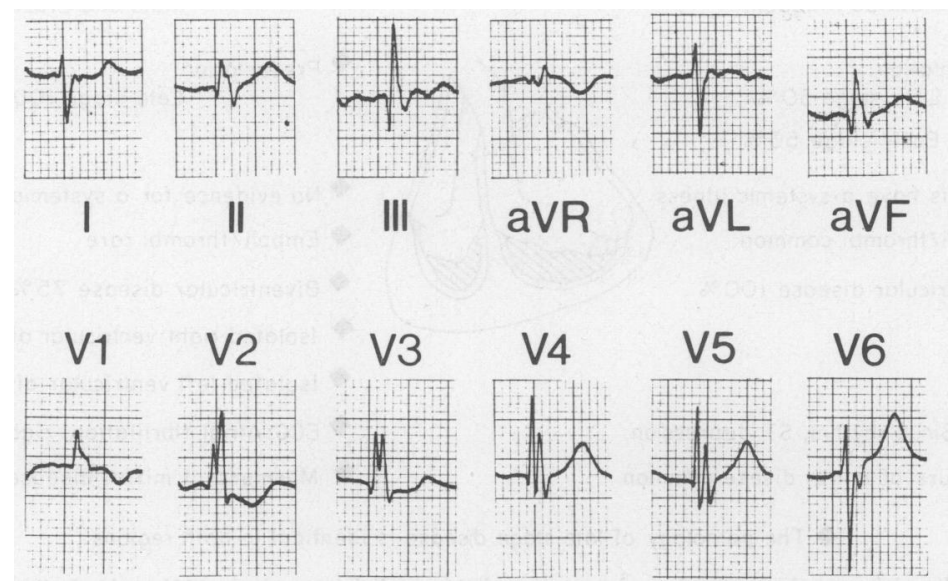

FIG. 4. Electrocardiogram of a child with tropical endomyocardial disease in India, showing atrial fibrillation, right axis deviation and ri舐
bundle branch block.

reported separately (Spry et al., 1983). The principal findings were that patients had markedly raised blood eosinophil counts, and that many of the eosinophils were degranulated. Three patients had high levels of serum IgM and IgE. Similar measurements in patients in India and Brazil were complicated by the common occurrence of parasitic infections in these areas. For this reason, comparisons were made in India between 28 patients with tropical endomyocardial disease, and age and sex matched control subjects. These showed that patients with tropical endomyocardial disease had blood eosinophil counts that were no higher than those in the control subjects. An important difference between these patients and those in the U.K. was that no degranulated eosinophils were found in their peripheral blood. The patients in India, and their controls, had raised immunoglobulin levels, but a higher proportion of the patients had markedly raised serum IgG, IgA, IgM and IgE. A larger number of patients in India had raised filarial antibody titres compared to controls.

\section{Discussion}

Although there is no pathological difference between late stage fibrotic endomyocardial disease in temperate and tropical climates (Brockington and Olsen, 1973), this study has clearly shown some important regional differences in the clinical presentation, cardiological features and laboratory abnormalities. The principal differences were in the sex incidence and age groups affected, and the absence of isolated left or right ventricular disease in the U.K. However, these studies show no fundamental differences in the nature or characteristics of endomyocar- dial disease as it affected the heart in either region? For this reason, these differences probably indicate variations in the underlying disease process, whicb leads to a final common cardiac pathology.

Patients in the U.K. all had one underlying disease:the hypereosinophilic syndrome. This did not appeaco to have been present in any of the patients studie in $^{\omega}$ India or Brazil, and none had a preceding histor suggesting that they had some variant of this syndrome. However, as there is strong clinical an $\bar{\Phi}$ experimental evidence to suggest that eosinophil\% themselves are involved in the development of eosinophilic endomyocardial disease (Spry, Tai and Davies, 1983), it is important to consider whethe $\$$ eosinophils might also have been involved in the development of tropical endomyocardial disease? Most of the Indian and Brazilian patients had at eosinophilia, even though they were in the late. fibrotic stage of the disease. They came from poo socio-economic groups and had a higher incidence of . parasitic diseases. The close association of endemi filariasis with tropical endomyocardial disease in South India is unlikely to be accidental. Patients witl tropical endomyocardial disease in this area had higher filarial antibody levels than controls, suggest? ing that they may have had repeated or more severes infections than controls who did not develop this disease. There does not appear to be an association of tropical endomyocardial disease in Brazil with single parasitic disease.

It is possible to explain a number of the differences between eosinophilic endomyocardial disease, iक temperate and tropical regions, by taking into ac $\stackrel{\infty}{=}$ count the nature of the underlying disease process. In temperate climates the hypereosinophilic syndromeo which is the principal cause for eosinophilic endomy 
ocardial disease, is a severe disorder leading to early presentation, episodes of acute endocarditis and rapid progression, over several months, to the late fibrotic lesions. On the other hand, in the tropics, parasites are the principal cause of an eosinophilia, which is so common that little attention is paid, unless the patient is unwell. Occasionally hypereosinophilia develops, as in tropical (filarial) eosinophilia (Spry and Kumaraswami, 1982). Most clinical problems associated with parasitic infections occur during childhood, when there is the highest incidence of infection, and adults usually have some degree of immunity or tolerance to their chronic parasite load. If eosinophil derived toxins are responsible for the development of tropical endomyocardial disease, this is likely to develop in a younger age group, over a more prolonged period, than in temperate climates where older male patients with the hypereosinophilic syndrome are most at risk from developing this disease. In the later stages of eosinophilic endomyocardial disease, the peripheral blood eosinophilia may decline or disappear. This may also occur in tropical endomyocardial disease, accounting for the normal blood eosinophil counts in a small proportion of these patients. This possibility emphasises the importance of detecting tropical endomyocardial disease in its early stages, so that a search can be made for possible underlying eosinuphilic disorders. As many of these respond well to treatment, it may be possible to prevent progression of tropical endomyocardial disease to its late stages.

It is concluded that, although a number of clinical and cardiological features of endomyocardial disease were found to be different in a temperate and two tropical regions, there were many similarities between them. For this reason we propose that they have a common pathogenesis linked to underlying eosinophilic disorders. It is suggested that differences are related to the various causes of eosinophilia in these regions, and the rate at which eosinophil granule toxins induce cardiac damage. It is therefore important to find methods for detecting tropical endomyocardial disease in its early stages before severe and life threatening damage has occurred.

\section{Acknowledgments}

This project is being supported by the Wellcome Trust and British Heart Foundation, and forms part of a multicentre project on endomyocardial disease which is being carried out under the auspices of the International Society and Federation of Cardiology. We are particularly grateful to Professor J.G. Goodwin. Dr Celia Oakley, Dr E.G.J. Olsen and Dr Bridget Ogilvie in London, Dr D.V. Nair and Dr George Jacob in Kottayam. Kerala. Dr S. Sadanandan and Dr Ramachambaram in Trivandrum and Professor Guimaraes in Bahia, Brazil for their advice and help in setting up this project. Further details of the clinical features and investigations in the patients reviewed here will be provided later in joint publications.

\section{References}

Brockington, I.F. \& OLSEN, E.G.J. (1973) Löffler's endocarditis and Davies' endomyocardial fibrosis. American Heart Journal, 85, 308.

Chaine, G., Davies, J., Kohner, E.M., Hawarth, S. \& Spry, C.J.F. (1982) Ophthalmological abnormalities in patients with the hypereosinophilic syndrome. Ophthalmology 89, 1348.

DAVIES, J.N.P. (1948) Endomyocardial necrosis. A heart disease of obscure aetiology in africans. $M D$ thesis, Bristol University.

Davies, J., Gibson, D.G., Foale, R., Heer, K., SPRY, C.J.F., OAKLEY, C.M. \& GOODWIN, J.F. (1982b) Echocardiographic features of eosinophilic endomyocardial disease. British Heart Journal 48, 434.

Davies, J., SPRy, C.J.F., SAPSford, R., Olsen, E.G.J., DuPerez, G., OAKLEY, C.M. \& GoOdWIN, J.F. (1983) Cardiovascular features of eleven patients with eosinophilic endomyocardial disease. Quarterly Journal of Medicine 52, 23.

GerbauX, A., DE BruX, J., BenNaceur, M. \& LenÉgre. J. (1956) L'endocardite pariétale fibroplastique avec éosinophilie sanguine (endocardite de Löffler). Bulletins et memoires de la Sociětè Medicale des Hopitaux du Paris, 72, 456.

IVE, F.A., WILLIS, A.J.P. \& IKEME, A.C. (1967) Endomyocardial fibrosis and filariasis. Quarterly Journal of Medicine, 36, 495.

OLSEN, E.G.J. \& SPRY, C.J.F. (1979) The pathogenesis of Löffler's endomyocardial disease and its relationship to endomyocardial fibrosis. Progress in Cardiology, 8, 281.

Patel, A.K., D'Arbela, P.G. \& Somers, K. (1977) Endomyocardial fibrosis and eosinophilia. British Heart Journal, 39, 238.

SPRY, C.J.F. (1982) The hypereosinophilic syndrome: clinical features, laboratory findings and treatment. Allergy 37, 539.

SPRY, C.J.F., Davies, J., TAI, P.C., OlSEN, E.G.J., OAKLeY, C.M. \& GoodwiN, J.F. (1982) Clinical features of 15 patients with the hypereosinophilic syndrome. Quarterly Journal of Medicine 52, 1.

SPRY, C.J.F. \& KumaraswamI. V. (1982) Tropical eosinophilia. Seminars in Haematology, 19, 107.

SPRY, C.J.F., TAI, P.C. \& DAVIES, J. (1983) The cardiotoxicity of eosinophils. Postgraduate Medical Journal, 59, 147. 